\title{
ON THE EXISTENCE OF WEAK SOLUTIONS FOR THE INITIAL-BOUNDARY VALUE PROBLEM IN THE JEFFREYS MODEL OF MOTION OF A VISCOELASTIC MEDIUM
}

\author{
D. A. VOROTNIKOV AND V. G. ZVYAGIN
}

Received 28 November 2003

This paper deals with the initial-boundary value problem for the system of motion equations of an incompressible viscoelastic medium with Jeffreys constitutive law in an arbitrary domain of two-dimensional or three-dimensional space. The existence of weak solutions of this problem is obtained.

\section{Introduction}

It is known $[5,7]$ that the motion of an incompressible medium with constant density $\rho=$ const is determined by the system of differential equations in the form of Cauchy

$$
\begin{gathered}
\rho\left(\frac{\partial u}{\partial t}+\sum_{i=1}^{n} u_{i} \frac{\partial u}{\partial x_{i}}\right)+\operatorname{grad} p=\operatorname{Div} \sigma+\rho f, \quad(t, x) \in(0, T) \times \Omega, \\
\operatorname{div} u=0, \quad(t, x) \in(0, T) \times \Omega .
\end{gathered}
$$

Here $u$ is the velocity vector, $p$ is the pressure function, $f$ is the body force, and $\sigma$ is the deviator of the stress tensor (all of them depend on a point $x$ of an arbitrary domain $\Omega$ in the space $\mathbb{R}^{n}, n=2,3$, and on a moment of time $t$ ). The gradient grad and the divergence div are taken with respect to the variable $x$. The divergence Div $\sigma$ of a tensor $\sigma$ is the vector with the coordinates $(\operatorname{Div} \sigma)_{j}=\sum_{i=1}^{n}\left(\partial \sigma_{i j} / \partial x_{i}\right)$.

Without loss of generality, we can consider the density $\rho$ to be equal to 1 .

The type of a medium is determined by the choice of the constitutive law between $\sigma$ and the strain velocity tensor $\mathscr{E}(u), \mathscr{E}(u)=\left(\mathscr{E}_{i j}(u)\right), \mathscr{E}_{i j}(u)=(1 / 2)\left(\partial u_{i} / \partial x_{j}+\partial u_{j} / \partial x_{i}\right)$. For instance, one class of mediums is connected with the Stokes conjecture that the deviator of the stress tensor in every point is completely determined by the strain velocity tensor in the same point at the same moment of time. It is the conception of linear- and nonlinear-viscous fluid [7].

However, this conception is not satisfactory for all incompressible media. In particular, it is not suitable for mediums "with memory": concrete, various polymers, the earth's crust, and so forth. To take into account the effects of memory one may introduce time 
derivatives into the constitutive law. When this method was used the models of Maxwell, Jeffreys, Oldroyd, and a lot of other models $[4,8,9]$ appeared.

In the present work we study the solvability in the weak sense of the initial-boundary value problem in the Jeffreys model [9] of motion of a viscoelastic medium in an arbitrary domain $\Omega \subset \mathbb{R}^{n}(n=2,3)$, which may also be unbounded. The corresponding constitutive law is

$$
\sigma+\lambda_{1} \frac{\mathrm{d}}{\mathrm{d} t} \sigma=2 \eta\left(\mathscr{E}+\lambda_{2} \frac{\mathrm{d}}{\mathrm{d} t} \mathscr{E}\right)
$$

Here $\eta$ is the viscosity of the medium, $\lambda_{1}$ is the relaxation time, and $\lambda_{2}$ is the retardation time, $0<\lambda_{2}<\lambda_{1}$.

The main result of this paper is the existence theorem of weak solutions for the initialboundary value problem for system (1.1)-(1.2) in an arbitrary domain $\Omega \subset \mathbb{R}^{n}(n=2,3)$.

We note that in a number of papers (see, e.g., $[1,2,6]$ ) the initial-boundary value problem was studied provided that the full derivative $\mathrm{d} / \mathrm{d} t$ was replaced by the partial derivative $\partial / \partial t$, what essentially narrows the class of mediums satisfying the model [8]. In [11] the Jeffreys relation (1.2) was considered without such linearizations, but at the expression of the stress tensor through the strain velocity tensor the regularization of the velocity field with the help of averaging on the spatial variable was used. The feature of this work is that here there is no such regularization.

The plan of the paper is as follows. In Section 2, the statement of the problem, describing the motion of the viscoelastic fluid, is presented and the basic notations are introduced. Then we introduce the concept of a weak solution of this problem and formulate the main existence theorem. In Section 3, the existence of solutions of an auxiliary problem, depending on several parameters, is proved with the help of a priori estimates and the Leray-Schauder degree theory. In Section 4, the passage to the limit as one of these parameters tends to zero is carried out. With the help of the obtained result, in Section 5, we prove the existence of a weak solution of the initial-boundary value problem for the Jeffreys model and estimate this solution.

\section{Statement of the problem and the main result}

2.1. Statement of the problem. Let $\Omega$ be an arbitrary domain in the space $\mathbb{R}^{n}, n=2,3$, which may also be unbounded.

Consider the following initial-boundary value problem, which describes the motion of an incompressible viscoelastic medium, corresponding to the Jeffreys model:

$$
\begin{gathered}
\frac{\partial u}{\partial t}+\sum_{i=1}^{n} u_{i} \frac{\partial u}{\partial x_{i}}+\operatorname{grad} p=\operatorname{Div} \sigma+f \\
\sigma+\lambda_{1}\left(\frac{\partial \sigma}{\partial t}+\sum_{i=1}^{n} u_{i} \frac{\partial \sigma}{\partial x_{i}}\right)=2 \eta\left(\mathscr{E}+\lambda_{2}\left(\frac{\partial \mathscr{E}}{\partial t}+\sum_{i=1}^{n} u_{i} \frac{\partial \mathscr{E}}{\partial x_{i}}\right)\right) \\
\operatorname{div} u=0 \\
\left.u\right|_{\partial \Omega}=0 \\
\left.u\right|_{t=0}=a,\left.\quad \sigma\right|_{t=0}=\sigma_{0} .
\end{gathered}
$$


2.2. Basic notations. We will use the following notations. Most of them are standard.

Denote by $\mathbb{R}^{n \times n}$ the space of matrices of order $n \times n$ with the following scalar product: for $A=\left(A_{i j}\right), B=\left(B_{i j}\right)$,

$$
(A, B)_{\mathbb{R}^{n \times n}}=\sum_{i, j=1}^{n} A_{i j} B_{i j},
$$

and denote by $\mathbb{R}_{S}^{n \times n}$ its subspace of symmetric matrices.

Denote by $\mathbb{R}^{n \times n \times n}$ the space of ordered collections of $n$ matrices of order $n \times n$ with the following scalar product: for $A=\left(A_{1}, \ldots, A_{n}\right), B=\left(B_{1}, \ldots, B_{n}\right)$,

$$
(A, B)_{\mathbb{R}^{n \times n \times n}}=\sum_{i=1}^{n}\left(A_{i}, B_{i}\right)_{\mathbb{R}^{n \times n}} .
$$

The symbol $\nabla u$ will stand for the Jacobi matrix of a vector function $u: \Omega \rightarrow \mathbb{R}^{n}$. The symbol $\nabla \tau$ will denote the ordered collection of the Jacobi matrices of the columns of a matrix function $\tau: \Omega \rightarrow \mathbb{R}^{n \times n}$.

Below, $E$ stands for one of the spaces $\mathbb{R}^{n}, \mathbb{R}^{n \times n}, \mathbb{R}_{S}^{n \times n}, \mathbb{R}^{n \times n \times n}$.

We will use the standard notations $L_{p}(\Omega, E), W_{p}^{m}(\Omega, E), H^{m}(\Omega, E)=W_{2}^{m}(\Omega, E)$, $H_{0}^{m}(\Omega, E)=W_{2}^{m}(\Omega, E)$ for Lebesgue and Sobolev spaces of functions with values in $E$. Sometimes we will write simply $L_{p}$ instead of $L_{p}(\Omega, E)$, and so forth, if it is clear from the context which space $E$ is used.

The scalar products in $L_{2}(\Omega, E)$ and $H^{1}(\Omega, E)$ may be given by the following bilinear forms:

$$
\begin{aligned}
(u, v) & =\int_{\Omega}(u(x), v(x))_{E} d x \\
(u, v)_{1} & =(u, v)+\sum_{i=1}^{n}\left(\frac{\partial u}{\partial x_{i}}, \frac{\partial v}{\partial x_{i}}\right)
\end{aligned}
$$

The norms in these spaces will be denoted by $\|\cdot\|$ and $\|\cdot\|_{1}$, respectively. The Euclid norm in $E$ will be denoted by $|\cdot|$. By the symbol $C_{0}^{\infty}(\Omega, E)$ we will denote the space of smooth functions with compact support in $\Omega$ and with values in $E$.

For brevity, we will denote by $C_{0}^{\infty}$ the space $C_{0}^{\infty}\left(\Omega, \mathbb{R}_{S}^{n \times n}\right)$. Denote by $\mathscr{V}$ the set $\{u \in$ $\left.C_{0}^{\infty}\left(\Omega, \mathbb{R}^{n}\right), \operatorname{div} u=0\right\}$. Let the symbols $H$ and $V$ denote the closures of $\mathscr{V}$ in $L_{2}\left(\Omega, \mathbb{R}^{n}\right)$ and $W_{2}^{1}\left(\Omega, \mathbb{R}^{n}\right)$, respectively. Following [10], we will identify the space $H$ and its conjugate space $H^{*}$. Therefore we have the embedding

$$
V \subset H \equiv H^{*} \subset V^{*}
$$

The value of a functional $v$ from $V^{*}$ or $H^{-m}(m=1,2)$ on an element $\varphi$ from $V$ or $H_{0}^{m}$, respectively, will be denoted as $\langle\nu, \varphi\rangle$.

The symbols $C([0, T] ; X), C_{w}([0, T] ; X), L_{2}(0, T ; X), \ldots$, will denote the Banach spaces of continuous, weakly continuous, quadratically integrable, and so forth functions on the segment $[0, T]$ with values in some Banach space $X$. 
The symbol $K_{i}(\cdot, \ldots, \cdot), i=0,1,2, \ldots$ will stand for positive constants, depending continuously on arguments, which will be enumerated. Other constants will be denoted by the symbol $C$.

2.3. Weak formulation of the problem. Let $f$ belong to the space $L_{2}\left(0, T ; V^{*}\right)$.

Definition 2.1. A pair of functions $(u, \sigma)$,

$$
\begin{gathered}
u \in L_{2}(0, T ; V) \bigcap C_{w}([0, T] ; H), \quad \frac{d u}{d t} \in L_{1}\left(0, T ; V^{*}\right), \\
\sigma \in L_{2}\left(0, T ; L_{2}\left(\Omega, \mathbb{R}_{S}^{N \times N}\right)\right) \bigcap C_{w}\left([0, T] ; H^{-1}\left(\Omega, \mathbb{R}_{S}^{N \times N}\right)\right),
\end{gathered}
$$

is a weak solution of problem (2.1)-(2.5) if it satisfies condition (2.5) and the equalities

$$
\begin{aligned}
& \frac{d}{d t}(u, \varphi)+(\sigma, \nabla \varphi)-\sum_{i=1}^{n}\left(u_{i} u, \frac{\partial \varphi}{\partial x_{i}}\right)=\langle f, \varphi\rangle, \\
& (\sigma, \Phi)+\lambda_{1} \frac{d}{d t}(\sigma, \Phi)-\lambda_{1} \sum_{i=1}^{n}\left(u_{i} \sigma, \frac{\partial \Phi}{\partial x_{i}}\right) \\
& =-2 \eta(u, \operatorname{Div} \Phi)-2 \eta \lambda_{2}\left(\frac{d}{d t}(u, \operatorname{Div} \Phi)+\sum_{i=1}^{n}\left(u_{i} \mathscr{E}(u), \frac{\partial \Phi}{\partial x_{i}}\right)\right)
\end{aligned}
$$

are true for all $\varphi \in \mathscr{V}$ and $\Phi \in C_{0}^{\infty}$ in the sense of distributions on $(0, T)$.

Remark 2.2. Equalities (2.11) appear from the following reasoning. Let $(u, \sigma, p)$ be a classical solution of problem (2.1)-(2.5). Taking the $L_{2}$-scalar product of equalities (2.1) and (2.2) with $\varphi \in \mathscr{V}$ and $\Phi \in C_{0}^{\infty}$, respectively, and integrating the obtained equalities by parts, we obtain identities (2.11).

2.4. The main result. The main result of this paper is the following.

Theorem 2.3. Given $f \in L_{2}\left(0, T ; V^{*}\right), a \in H, \sigma_{0} \in W_{2}^{-1}\left(\Omega, \mathbb{R}_{S}^{n \times n}\right), \sigma_{0}-2 \eta\left(\lambda_{2} / \lambda_{1}\right) \mathscr{E}(a) \in$ $L_{2}\left(\Omega, \mathbb{R}_{S}^{n \times n}\right)$, there exists a weak solution of problem (2.1)-(2.5) in class (2.10).

\section{Auxiliary problem}

Before proving Theorem 2.3, we study an auxiliary problem. We begin with an equivalent transformation of system (2.11). Denote $\mu_{1}=\eta\left(\lambda_{2} / \lambda_{1}\right), \mu_{2}=\left(\eta-\mu_{1}\right) / \lambda_{1}$, and $\tau=\sigma-$ $2 \mu_{1} \mathscr{E}(u)$. Then we can rewrite $(2.11)$ as follows:

$$
\begin{gathered}
\frac{d}{d t}(\tau, \Phi)+\frac{1}{\lambda_{1}}(\tau, \Phi)-\sum_{i=1}^{n}\left(u_{i} \tau, \frac{\partial \Phi}{\partial x_{i}}\right)+2 \mu_{2}(u, \operatorname{Div} \Phi)=0, \\
\frac{d}{d t}(u, \varphi)-\sum_{i=1}^{n}\left(u_{i} u, \frac{\partial \varphi}{\partial x_{i}}\right)+\mu_{1}(\nabla u, \nabla \varphi)+(\tau, \nabla \varphi)=\langle f, \varphi\rangle
\end{gathered}
$$

for all $\varphi \in \mathscr{V}$ and $\Phi \in C_{0}^{\infty}$. 
Consider the following auxiliary problem:

$$
\begin{gathered}
\frac{d}{d t}(\tau, \Phi)+\frac{1}{\lambda_{1}}(\tau, \Phi)-\xi \sum_{i=1}^{n}\left(\frac{u_{i} \tau}{1+\delta\left(|\tau|^{2} / 2 \mu_{2}+|u|^{2}\right)}, \frac{\partial \Phi}{\partial x_{i}}\right)+2 \mu_{2} \xi(u, \operatorname{Div} \Phi)+\frac{\varepsilon}{\lambda_{1}}(\nabla \tau, \nabla \Phi)=0 \\
\frac{d}{d t}(u, \varphi)-\xi \sum_{i=0}^{n}\left(\frac{u_{i} u}{1+\delta\left(|\tau|^{2} / 2 \mu_{2}+|u|^{2}\right)}, \frac{\partial \varphi}{\partial x_{i}}\right)+\mu_{1}(\nabla u, \nabla \varphi)+\xi(\tau, \nabla \varphi)=\langle f, \varphi\rangle
\end{gathered}
$$

for all $\varphi \in V, \Phi \in H_{0}^{1}$ a.e. in $(0, T)$;

$$
\left.u\right|_{t=0}=a,\left.\quad \tau\right|_{t=0}=\tau_{0} .
$$

The numbers $\delta>0,0 \leq \xi \leq 1,0<\varepsilon \leq 1$ are parameters.

We introduce the following spaces:

$$
\begin{aligned}
W & =\left\{u \in L_{2}(0, T ; V), \frac{d u}{d t} \in L_{2}\left(0, T ; V^{*}\right)\right\} \\
W_{M} & =\left\{\tau \in L_{2}\left(0, T ; H_{0}^{1}\left(\Omega, \mathbb{R}_{S}^{n \times n}\right)\right), \frac{d \tau}{d t} \in L_{2}\left(0, T ; H^{-1}\left(\Omega, \mathbb{R}_{S}^{n \times n}\right)\right)\right\}
\end{aligned}
$$

with natural intersection norms.

It follows from [10, Chapter III, Theorem 1.2] that $W$ and $W_{M}$ are embedded into $C([0, T] ; H)$ and $C\left([0, T] ; L_{2}\right)$, respectively. If $\Omega$ is bounded, (see [10, Chapter III, Theorem 2.1] and [10, Chapter II, Theorem 1.1]), the embeddings $W$ into $L_{2}(0, T ; H)$ and $W_{M}$ into $L_{2}\left(0, T ; L_{2}\right)$ are compact.

Lemma 3.1. Let $a \in H, \tau_{0} \in L_{2}, f \in L_{2}\left(0, T ; V^{*}\right)$, and let a pair $\left(u \in W, \tau \in W_{M}\right)$ be a solution of problem (3.3)-(3.5). Then the following estimate holds:

$$
\begin{aligned}
\max _{t \in[0, T]}(\|u\|(t)+\|\tau\|(t))+\int_{0}^{T}\|u\|_{1}^{2}(t) d t+\varepsilon \int_{0}^{T}\|\tau\|_{1}^{2}(t) d t \\
\leq K_{0}\left(\|a\|,\left\|\tau_{0}\right\|,\|f\|_{L_{2}\left(0, T ; V^{*}\right)}\right)
\end{aligned}
$$

where $K_{0}$ does not depend on $\Omega, \varepsilon, \delta, \xi$.

Proof. First we show that the following identities hold a.e. in $(0, T)$ :

$$
\begin{gathered}
(\tau, \nabla u)+(u, \operatorname{Div} \tau)=0 \\
\sum_{i=1}^{n}\left(\frac{u_{i} u}{1+\delta\left(|\tau|^{2} / 2 \mu_{2}+|u|^{2}\right)}, \frac{\partial u}{\partial x_{i}}\right)+\frac{1}{2 \mu_{2}}\left(\frac{u_{i} \tau}{1+\delta\left(|\tau|^{2} / 2 \mu_{2}+|u|^{2}\right)}, \frac{\partial \tau}{\partial x_{i}}\right)=0
\end{gathered}
$$


In fact, from Green's formula we have

$$
\sum_{i, j=1}^{n}\left[\left(\tau_{i j}, \frac{\partial u_{i}}{\partial x_{j}}\right)+\left(u_{i}, \frac{\partial \tau_{i j}}{\partial x_{j}}\right)\right]=0
$$

and it is the same as (3.8). We transform the left part of (3.9) as follows:

$$
\begin{array}{r}
\frac{1}{2}\left(\sum_{i=1}^{n} \frac{u_{i}}{1+\delta\left(|\tau|^{2} / 2 \mu_{2}+|u|^{2}\right)}, \frac{\partial|u|^{2}}{\partial x_{i}}+\frac{1}{2 \mu_{2}} \frac{\partial|\tau|^{2}}{\partial x_{i}}\right) \\
=\frac{1}{2 \delta} \sum_{i=1}^{n}\left(u_{i}, \frac{\partial}{\partial x_{i}} \ln \left(1+\delta\left(\frac{|\tau|^{2}}{2 \mu_{2}}+|u|^{2}\right)\right)\right) .
\end{array}
$$

Since $u(t) \in V$ for a.a. $t$, from the formula of integration by parts it follows that the last expression is equal to zero.

It appears from [10, Chapter III, Lemma 1.1] that

$$
\left\langle\frac{d u}{d t}, \varphi\right\rangle=\frac{d}{d t}(u, \varphi), \quad\left\langle\frac{d \tau}{d t}, \Phi\right\rangle=\frac{d}{d t}(\tau, \Phi)
$$

Therefore

$$
\left.\frac{d}{d t}(u, \varphi)\right|_{\varphi=u(t)}=\left\langle\frac{d u(t)}{d t}, u(t)\right\rangle=\frac{1}{2} \frac{d}{d t}(u(t), u(t))
$$

Analogously

$$
\left.\frac{d}{d t}(\tau, \Phi)\right|_{\Phi=\tau(t)}=\frac{1}{2} \frac{d}{d t}(\tau, \tau)
$$

Putting $\Phi=\tau(t) / 2 \mu_{2}$ in (3.3) and $\varphi=u(t)$ in (3.4) for a.a. $t \in[0, T]$, adding the results, and taking into account (3.8) and (3.9), we obtain

$$
\frac{1}{2} \frac{d}{d t}(u, u)+\frac{1}{4 \mu_{2}} \frac{d}{d t}(\tau, \tau)+\mu_{1}(\nabla u, \nabla u)+\frac{1}{2 \lambda_{1} \mu_{2}}(\tau, \tau)+\frac{\varepsilon}{2 \lambda_{1} \mu_{2}}(\nabla \tau, \nabla \tau)=\langle f, u\rangle .
$$

Integrate this equality from 0 to $t$ :

$$
\begin{aligned}
\frac{1}{2}\|u\|^{2}(t) & +\frac{1}{4 \mu_{2}}\|\tau\|^{2}(t)+\int_{0}^{t} \frac{1-\varepsilon}{2 \lambda_{1} \mu_{2}}\|\tau\|^{2} d s+\int_{0}^{t} \frac{\varepsilon}{2 \lambda_{1} \mu_{2}}\|\tau\|_{1}^{2} d s+\int_{0}^{t} \mu_{1}\left(\|u\|_{1}^{2}-\|u\|^{2}\right) d s \\
& \leq \frac{1}{2}\|a\|^{2}+\frac{1}{4 \mu_{2}}\left\|\tau_{0}\right\|^{2}+\int_{0}^{t}\|f\|_{V^{*}}\|u\|_{1} d s .
\end{aligned}
$$


Take the maxima on the segment $[0, T]$ of the left and the right parts:

$$
\begin{aligned}
\max _{t \in[0, T]} & \left(\frac{1}{2}\|u\|^{2}(t)+\frac{1}{4 \mu_{2}}\|\tau\|^{2}(t)\right)+\int_{0}^{T} \frac{1-\varepsilon}{2 \lambda_{1} \mu_{2}}\|\tau\|^{2} d t+\int_{0}^{T} \frac{\varepsilon}{2 \lambda_{1} \mu_{2}}\|\tau\|_{1}^{2} d t \\
& +\int_{0}^{T} \mu_{1}\left(\|u\|_{1}^{2}-\|u\|^{2}\right) d t \leq \frac{1}{2}\|a\|^{2}+\frac{1}{4 \mu_{2}}\left\|\tau_{0}\right\|^{2}+\int_{0}^{T}\|f\|_{V^{*}}\|u\|_{1} d t .
\end{aligned}
$$

Note that the following inequality is valid:

$$
\max _{t \in[0, T]} \frac{1}{4}\|u\|^{2}(t)+\int_{0}^{T} \mu_{1}\left(\|u\|_{1}^{2} d t-\|u\|^{2}\right) \geq \gamma \int_{0}^{T}\|u\|_{1}^{2} d t
$$

where $\gamma=\min \left(1 / 4 T, \mu_{1}\right)$.

For its proof it is enough to add the inequalities

$$
\begin{gathered}
\max _{t \in[0, T]} \frac{1}{4}\|u\|^{2}(t) \geq \frac{1}{4 T} \int_{0}^{T}\|u\|^{2} d t \geq \gamma \int_{0}^{T}\|u\|^{2} d t \\
\int_{0}^{T} \mu_{1}\left(\|u\|_{1}^{2}-\|u\|^{2}\right) d t \geq \gamma \int_{0}^{T}\left(\|u\|_{1}^{2}-\|u\|^{2}\right) d t .
\end{gathered}
$$

Now, from (3.17) and (3.18) we have

$$
\gamma \int_{0}^{T}\|u\|_{1}^{2} d t \leq \frac{1}{2}\|a\|^{2}+\frac{1}{4 \mu_{2}}\left\|\tau_{0}\right\|^{2}+\|f\|_{L_{2}\left(0, T ; V^{*}\right)}\left(\int_{0}^{T}\|u\|_{1}^{2} d t\right)^{1 / 2} .
$$

This yields $\left(\int_{0}^{T}\|u\|_{1}^{2} d t\right)^{1 / 2} \leq y_{2}$, where $y_{2}$ is the greater root of the quadratic equation

$$
\gamma y^{2}=\frac{1}{2}\|a\|^{2}+\frac{1}{4 \mu_{2}}\left\|\tau_{0}\right\|^{2}+\|f\|_{L_{2}\left(0, T ; V^{*}\right)} y .
$$

Then, from (3.17) and (3.18), it follows that

$$
\begin{aligned}
\max _{t \in[0, T]} & \left(\frac{1}{4}\|u\|^{2}(t)+\frac{1}{4 \mu_{2}}\|\tau\|^{2}(t)\right)+\gamma \int_{0}^{T}\|u\|_{1}^{2} d t+\frac{\varepsilon}{2 \lambda_{1} \mu_{2}} \int_{0}^{T}\|\tau\|_{1}^{2} d t \\
& \leq \frac{1}{2}\|a\|^{2}+\frac{1}{4 \mu_{2}}\left\|\tau_{0}\right\|^{2}+\|f\|_{L_{2}\left(0, T ; V^{*}\right)} y_{2},
\end{aligned}
$$

which yields the statement of the lemma.

Theorem 3.2. Let $\Omega$ be bounded and let $a, \tau_{0}, f$ satisfy the conditions of Lemma 3.1. Then problem (3.3)-(3.5) possesses a solution $u \in W, \tau \in W_{M}$. 
Proof. Introduce auxiliary operators by the following formulas (in these formulas $\varphi$ and $\Phi$ are arbitrary elements of $V$ and $H_{0}^{1}\left(\Omega, \mathbb{R}_{S}^{n \times n}\right)$, resp.):

$$
\begin{aligned}
& N_{1}: W_{M} \longrightarrow L_{2}\left(0, T ; V^{*}\right), \quad\left\langle N_{1}(\tau), \varphi\right\rangle=(\tau, \nabla \varphi), \\
& N_{2}: W \longrightarrow L_{2}\left(0, T ; H^{-1}\right), \quad\left\langle N_{2}(u), \Phi\right\rangle=2 \mu_{2}(u, \operatorname{Div} \Phi), \\
& K_{\delta}: W \times W_{M} \longrightarrow L_{2}\left(0, T ; V^{*}\right), \\
& \left\langle K_{\delta}(u, \tau), \varphi\right\rangle=-\sum_{i=1}^{n}\left(\frac{u_{i} u}{1+\delta\left(|u|^{2}+|\tau|^{2} / 2 \mu_{2}\right)}, \frac{\partial \varphi}{\partial x_{i}}\right), \\
& \tilde{K}_{\delta}: W \times W_{M} \longrightarrow L_{2}\left(0, T ; H^{-1}\right), \\
& \left\langle\tilde{K}_{\delta}(u, \tau), \Phi\right\rangle=-\sum_{i=1}^{n}\left(\frac{u_{i} \tau}{1+\delta\left(|u|^{2}+|\tau|^{2} / 2 \mu_{2}\right)}, \frac{\partial \Phi}{\partial x_{i}}\right), \\
& A: W \longrightarrow L_{2}\left(0, T ; V^{*}\right), \\
& \langle A(u), \varphi\rangle=\mu_{1}(\nabla u, \nabla \varphi), \\
& A_{\varepsilon}: W_{M} \longrightarrow L_{2}\left(0, T ; H^{-1}\right), \\
& \left\langle A_{\varepsilon}(\tau), \Phi\right\rangle=\varepsilon(\nabla \tau, \nabla \Phi)+\frac{1}{\lambda_{1}}(\tau, \Phi), \\
& \tilde{A}: W \times W_{M} \longrightarrow L_{2}\left(0, T ; V^{*}\right) \times L_{2}\left(0, T ; H^{-1}\right) \times H \times L_{2}, \\
& \tilde{A}(u, \tau)=\left(\frac{d u}{d t}+A(u), \frac{d \tau}{d t}+A_{\varepsilon}(\tau),\left.u\right|_{t=0},\left.\tau\right|_{t=0}\right), \\
& Q: W \times W_{M} \longrightarrow L_{2}\left(0, T ; V^{*}\right) \times L_{2}\left(0, T ; H^{-1}\right) \times H \times L_{2}, \\
& Q(u, \tau)=\left(K_{\delta}(u, \tau)+N_{1}(\tau), \tilde{K}_{\delta}(u, \tau)+N_{2}(u), 0,0\right) .
\end{aligned}
$$

Then problem (3.3)-(3.5) is equivalent to the operator equation

$$
\tilde{A}(u, \tau)+\xi Q(u, \tau)=\left(f, 0, a, \tau_{0}\right) .
$$

The operator $\tilde{A}$ is invertible by [3, Chapter VI, Theorem 1.1]. Moreover, since the embeddings $W$ into $L_{2}(0, T ; H)$ and $W_{M}$ into $L_{2}\left(0, T ; L_{2}\right)$ are compact, the operators $N_{1}$, $N_{2}$ are compact. The operators $K_{\delta}$ and $\tilde{K}_{\delta}$ are compact, which may be shown as in [2, Theorem 2.2]. Hence, the operator $Q$ is also compact.

Rewrite (3.24) as

$$
(u, \tau)-\xi \tilde{A}^{-1} Q(u, \tau)=\tilde{A}^{-1}\left(f, 0, a, \tau_{0}\right) .
$$

By Lemma 3.1 equation (3.25) has no solutions on the boundary of a sufficiently large ball $B$ in $W \times W_{M}$, independent on $\xi, \delta$, $\varepsilon$. Without loss of generality, $a_{0}=\tilde{A}^{-1}\left(f, 0, a, \tau_{0}\right)$ belongs to this ball. Then $\operatorname{deg}_{\mathrm{LS}}\left(I-\xi \tilde{A}^{-1} Q, B, a_{0}\right)$, the Leray-Schauder degree of the map $I-\xi \tilde{A}^{-1} Q$ on the ball $B$ with respect to the point $a_{0}$, is defined, where $I$ is the identity 
operator. By the homotopic invariance property of the degree we have

$$
\operatorname{deg}_{\mathrm{LS}}\left(I-\xi \tilde{A}^{-1} Q, B, a_{0}\right)=\operatorname{deg}_{\mathrm{LS}}\left(I, B, a_{0}\right)=1 .
$$

Hence, (3.25) (and therefore, problem (3.3)-(3.5)) has a solution in $B$ for every $\xi$.

We need the following estimates on the time derivatives of the solutions of problem (3.3)-(3.5).

LEMMA 3.3. In the conditions of the previous theorem the following estimates of the solutions are valid:

$$
\begin{gathered}
\left\|\frac{d u}{d t}\right\|_{L_{1}\left(0, T ; V^{*}\right)} \leq K_{1}\left(\|a\|,\left\|\tau_{0}\right\|,\|f\|_{L_{2}\left(0, T ; V^{*}\right)}\right), \\
\left\|\frac{d \tau}{d t}\right\|_{L_{1}\left(0, T ; H^{-1}\right)} \leq K_{2}\left(\|a\|,\left\|\tau_{0}\right\|,\|f\|_{\left.L_{2}\left(0, T ; V^{*}\right), \mathcal{E}\right)},\right.
\end{gathered}
$$

where $K_{1}, K_{2}$ do not depend on $\Omega, \delta$, $\xi$, and $K_{1}$ also does not depend on $\varepsilon$.

Proof. From (3.4) we have

$$
\begin{aligned}
\int_{0}^{T}\left|\frac{d}{d t}(u, \varphi)\right| d t \leq \int_{0}^{T} & \left|\xi \sum_{i=1}^{n}\left(\frac{u_{i} u}{1+\delta\left(|\tau|^{2} / 2 \mu_{2}+|u|^{2}\right)}, \frac{\partial \varphi}{\partial x_{i}}\right)\right| \\
& +\left|\mu_{1}(\nabla u, \nabla \varphi)\right|+|\xi(\tau, \nabla \varphi)|+|\langle f, \varphi\rangle| d t .
\end{aligned}
$$

Applying Hölder's inequality and estimate (3.7), and taking into account the embed$\operatorname{ding} V \subset L_{4}$ (provided $\left.N=2,3\right)$ and the inequality $\xi /\left(1+\delta\left(|\tau|^{2} / 2 \mu_{2}+|u|^{2}\right)\right) \leq 1$, we see that the right part does not exceed

$$
\begin{aligned}
\|\varphi\|_{V} & \int_{0}^{T}\left(\|u(t)\|_{L_{4}}^{2}+\mu_{1}\|u(t)\|_{1}+\|\tau(t)\|+\|f(t)\|_{V^{*}}\right) d t \\
& \leq C\|\varphi\|_{V}\left(1+\|u\|_{L_{2}(0, T ; V)}^{2}+\|\tau\|_{L_{\infty}\left(0, T ; L_{2}\right)}+\|f\|_{L_{2}\left(0, T ; V^{*}\right)}\right) \\
& \leq K_{1}\left(\|a\|,\left\|\tau_{0}\right\|,\|f\|_{L_{2}\left(0, T ; V^{*}\right)}\right)\|\varphi\|_{V} .
\end{aligned}
$$

These estimates and (3.12) yield (3.27).

Analogously, from (3.3), one obtains (3.28).

\section{Passage to the limit}

Consider one more auxiliary system

$$
\begin{gathered}
\frac{d}{d t}(\tau, \Phi)+\frac{1}{\lambda_{1}}(\tau, \Phi)-\sum_{i=1}^{n}\left(u_{i} \tau, \frac{\partial \Phi}{\partial x_{i}}\right)+2 \mu_{2}(u, \operatorname{Div} \Phi)+\frac{\varepsilon}{\lambda_{1}}(\nabla \tau, \nabla \Phi)=0, \\
\frac{d}{d t}(u, \varphi)-\sum_{i=1}^{n}\left(u_{i} u, \frac{\partial \varphi}{\partial x_{i}}\right)+\mu_{1}(\nabla u, \nabla \varphi)+(\tau, \nabla \varphi)=\langle f, \varphi\rangle,
\end{gathered}
$$

for all $\varphi \in V, \Phi \in H_{0}^{1}$ a.e. in $(0, T), 0<\varepsilon \leq 1$. 
Theorem 4.1. Let $\Omega, a, \tau_{0}, f$ satisfy the conditions of Theorem 3.2. Then problem (4.1), (3.5) possesses a solution in the class

$$
\begin{array}{cl}
u \in L_{2}(0, T ; V), & \tau \in L_{2}\left(0, T ; H_{0}^{1}\right), \\
\frac{d u}{d t} \in L_{1}\left(0, T ; V^{*}\right), & \frac{d \tau}{d t} \in L_{1}\left(0, T ; H^{-1}\right),
\end{array}
$$

which satisfies estimates (3.27), (3.28), and

$$
\begin{aligned}
\operatorname{vrai} \max _{t \in[0, T]} & (\|u\|(t)+\|\tau\|(t))+\int_{0}^{T}\|u\|_{1}^{2}(t) d t+\varepsilon \int_{0}^{T}\|\tau\|_{1}^{2}(t) d t \\
& \leq K_{0}\left(\|a\|,\left\|\tau_{0}\right\|,\|f\|_{L_{2}\left(0, T ; V^{*}\right)}\right) .
\end{aligned}
$$

Proof. Consider problems (3.3)-(3.5) with $\xi=1$ and $\delta=1 / m, m=1,2, \ldots$. By Theorem 3.2 there exist solutions $\left(u_{m}, \tau_{m}\right)$ of these problems. Taking into account estimate (3.7), without loss of generality, we may assume that

$$
\begin{array}{ll}
u_{m} \longrightarrow u_{*} & \text { weakly in } L_{2}(0, T ; V), \\
u_{m} \longrightarrow u_{*} & * \text {-weakly in } L_{\infty}(0, T ; H), \\
\tau_{m} \longrightarrow \tau_{*} & \text { weakly in } L_{2}\left(0, T ; H_{0}^{1}\right), \\
\tau_{m} \longrightarrow \tau_{*} & * \text {-weakly in } L_{\infty}\left(0, T ; L_{2}\right),
\end{array}
$$

as $m \rightarrow \infty$.

By Lemma 3.3 the sequence $\left\{d u_{m} / d t\right\}$ is bounded in $L_{1}\left(0, T ; V^{*}\right)$, and the sequence $\left\{d \tau_{m} / d t\right\}$ is bounded in $L_{1}\left(0, T ; H^{-1}\right)$. Then, by the compactness theorem (see [10, Chapter III, Theorem 2.3]),

$$
\begin{array}{ll}
u_{m} \longrightarrow u_{*} & \text { strongly in } L_{2}(0, T ; H), \\
\tau_{m} \longrightarrow \tau_{*} & \text { strongly in } L_{2}\left(0, T ; L_{2}\right),
\end{array}
$$

and we may assume that

$$
\begin{array}{ll}
u_{m}(t)(x) \longrightarrow u_{*}(t)(x) & \text { a.e. in }(0, T) \times \Omega, \\
\tau_{m}(t)(x) \longrightarrow \tau_{*}(t)(x) & \text { a.e. in }(0, T) \times \Omega .
\end{array}
$$

It is obvious that estimate $(4.3)$ is valid for $\left(u_{*}, \tau_{*}\right)$.

Substitute $\left(u_{m}, \tau_{m}\right)$ in equalities (3.3) and (3.4) with $\delta=1 / m, \xi=1$. Taking the scalar product of these equalities in $L_{2}(0, T)$ with a smooth scalar function $\psi(t), \psi(T)=0$, and 
integrating by parts the first terms, we obtain

$$
\begin{aligned}
& -\int_{0}^{T}\left(\tau_{m}, \Phi \psi^{\prime}(t)\right) d t+\int_{0}^{T}\left(\frac{1}{\lambda_{1}}\left(\tau_{m}, \psi \Phi\right)-\sum_{i=1}^{n}\left(\frac{\left(u_{m}\right)_{i} \tau_{m}}{1+(1 / m)\left(\left|\tau_{m}\right|^{2} / 2 \mu_{2}+\left|u_{m}\right|^{2}\right)}, \psi \frac{\partial \Phi}{\partial x_{i}}\right)\right. \\
& \left.\quad+2 \mu_{2}\left(u_{m}, \psi \operatorname{Div} \Phi\right)+\frac{\varepsilon}{\lambda_{1}}\left(\nabla \tau_{m}, \psi \nabla \Phi\right)\right) d t=\left(\tau_{0}, \Phi\right) \psi(0), \\
& -\int_{0}^{T}\left(u_{m}, \varphi \psi^{\prime}(t)\right) d t \\
& +\int_{0}^{T}\left(\mu_{1}\left(\nabla u_{m}, \psi \nabla \varphi\right)-\sum_{i=1}^{n}\left(\frac{\left(u_{m}\right)_{i} u_{m}}{1+(1 / m)\left(\left|\tau_{m}\right|^{2} / 2 \mu_{2}+\left|u_{m}\right|^{2}\right)}, \psi \frac{\partial \Phi}{\partial x_{i}}\right)+\left(\tau_{m}, \psi \nabla \varphi\right)\right) d t \\
& =\int_{0}^{T}\langle f, \varphi \psi\rangle d t+(a, \varphi) \psi(0) .
\end{aligned}
$$

As in [2, page 42], we may see that

$$
\begin{aligned}
& \int_{0}^{T} \sum_{i=1}^{n}\left(\frac{\left(u_{m}\right)_{i} \tau_{m}}{1+(1 / m)\left(\left|\tau_{m}\right|^{2} / 2 \mu_{2}+\left|u_{m}\right|^{2}\right)}, \psi \frac{\partial \Phi}{\partial x_{i}}\right) d t \rightarrow \int_{i=1}^{T} \sum_{i=1}^{n}\left(\left(u_{*}\right)_{i} \tau_{*}, \psi \frac{\partial \Phi}{\partial x_{i}}\right) d t \\
& \int_{0}^{T} \sum_{i=1}^{n}\left(\frac{\left(u_{m}\right)_{i} u_{m}}{1+(1 / m)\left(\left|\tau_{m}\right|^{2} / 2 \mu_{2}+\left|u_{m}\right|^{2}\right)}, \psi \frac{\partial \varphi}{\partial x_{i}}\right) d t \longrightarrow \int_{0}^{T} \sum_{i=1}^{n}\left(\left(u_{*}\right)_{i} u_{*}, \psi \frac{\partial \varphi}{\partial x_{i}}\right) d t .
\end{aligned}
$$

Passing to the limit in (4.7) as $m \rightarrow \infty$, we have

$$
\begin{gathered}
-\int_{0}^{T}\left(\tau_{*}, \Phi \psi^{\prime}(t)\right) d t+\int_{0}^{T}\left(\frac{1}{\lambda_{1}}\left(\tau_{*}, \psi \Phi\right)-\sum_{i=1}^{n}\left(\left(u_{*}\right)_{i} \tau_{*}, \psi \frac{\partial \Phi}{\partial x_{i}}\right)\right. \\
\left.+2 \mu_{2}\left(u_{*}, \psi \operatorname{Div} \Phi\right)+\frac{\varepsilon}{\lambda_{1}}\left(\nabla \tau_{*}, \psi \nabla \Phi\right)\right) d t=\left(\tau_{0}, \Phi\right) \psi(0), \\
-\int_{0}^{T}\left(u_{*}, \Phi \psi^{\prime}(t)\right) d t+\int_{0}^{T}\left(\mu_{1}\left(\nabla u_{*}, \psi \nabla \Phi\right)-\sum_{i=1}^{n}\left(\left(u_{*}\right)_{i} u_{*}, \psi \frac{\partial \Phi}{\partial x_{i}}\right)\right. \\
\left.+\left(\tau_{*}, \psi \nabla \varphi\right)\right) d t=\int_{0}^{T}\langle f, \varphi \psi\rangle d t+(a, \varphi) \psi(0) .
\end{gathered}
$$

Since it has been carried out, in particular, for every $\psi \in C_{0}^{\infty}(0, T)$, the function $\left(u_{*}, \tau_{*}\right)$ satisfies (4.1) in the sense of distributions on $(0, T)$.

Substitute $\left(u_{*}, \tau_{*}\right)$ into equalities (4.1). Since all terms in the obtained equalities are integrable on $(0, T)$, these equalities are valid a.e. on $(0, T)$. Taking the scalar product of these equalities in $L_{2}(0, T)$ with a smooth scalar function $\psi(t), \psi(T)=0, \psi(0) \neq 0$, and 
comparing the result with (4.9), we see that

$$
\begin{aligned}
& \left(\left.u_{*}\right|_{t=0}, \varphi\right) \psi(0)=(a, \varphi) \psi(0), \\
& \left(\left.\tau_{*}\right|_{t=0}, \Phi\right) \psi(0)=\left(\tau_{0}, \Phi\right) \psi(0) .
\end{aligned}
$$

Since $\Phi$ and $\varphi$ are arbitrary, $u_{*}$ and $\tau_{*}$ satisfy (3.5). Repeating the proof of Lemma 3.3 with $\delta=0, \xi=1$, we see that the solutions of problem (4.1), (3.5) satisfy estimates (3.27), (3.28). Thus, $\left(u_{*}, \tau_{*}\right)$ is the desirable solution.

\section{Existence of a weak solution for the Jeffreys model and its estimation}

First we will prove a statement from which Theorem 2.3 immediately follows.

Theorem 5.1. Given $f \in L_{2}\left(0, T ; V^{*}\right), a \in H, \tau_{0} \in L_{2}$, there exists a pair offunctions $(u, \tau)$,

$$
\begin{gathered}
u \in L_{2}(0, T ; V) \bigcap L_{\infty}(0, T ; H) \bigcap C_{w}([0, T], H), \quad \frac{d u}{d t} \in L_{1}\left(0, T ; V^{*}\right), \\
\tau \in L_{\infty}\left(0, T ; L_{2}\right) \bigcap C_{w}\left([0, T], L_{2}\right), \quad \frac{d \tau}{d t} \in L_{2}\left(0, T ; H^{-2}\right),
\end{gathered}
$$

satisfying (3.1), (3.2) a.e. in (0,T), the initial condition (3.5), and the estimate

$$
\begin{aligned}
\|u\|_{L_{2}(0, T ; V)} & +\|u\|_{L_{\infty}(0, T ; H)}+\left\|\frac{d u}{d t}\right\|_{L_{1}\left(0, T ; V^{*}\right)}+\|\tau\|_{L_{\infty}\left(0, T ; L_{2}\right)}+\left\|\frac{d \tau}{d t}\right\|_{L_{2}\left(0, T ; H^{-2}\right)} \\
& \leq K_{3}\left(\|a\|,\left\|\tau_{0}\right\|,\|f\|_{L_{2}\left(0, T ; V^{*}\right)}\right)
\end{aligned}
$$

where $K_{3}$ does not depend on $\Omega$.

Proof. Denote by $\Omega_{m}$ the intersection of $\Omega$ with the ball $B_{m}$ of radius $m$ with the center in the origin in the space $\mathbb{R}^{n}, m=1,2, \ldots$. From the definition of the space $H$ it follows that there exists a sequence $a_{m} \in C_{0}^{\infty}(\Omega), \operatorname{div} a_{m}=0$, supp $a_{m} \subset \Omega_{m}$, which converges to $a$ in $L_{2}(\Omega)$. Without loss of generality, $\left\|a_{m}\right\| \leq\|a\|$. Consider, for every $m$ on $\Omega_{m}$, problem (4.1) with $\varepsilon=1 / m$ and the following initial condition:

$$
\left.u\right|_{t=0}=a_{m},\left.\quad \tau\right|_{t=0}=\left.\tau_{0}\right|_{\Omega_{m}} .
$$

By Theorem 4.1 there exists a solution $\left(u_{m}, \tau_{m}\right)$ of this problem. Denote by $\tilde{u}_{m}$ and $\tilde{\tau}_{m}$ the functions which coincide with $u_{m}$ and $\tau_{m}$, respectively, in $\Omega_{m}$ and are identically zero in $\Omega \backslash \Omega_{m}$.

As in the proof of Theorem 4.1, without loss of generality, we may assume that

$$
\begin{aligned}
& \tilde{u}_{m} \longrightarrow u_{*} \quad \text { weakly in } L_{2}(0, T ; V), \\
& \tilde{u}_{m} \longrightarrow u_{*} \quad{ }^{*} \text {-weakly in } L_{\infty}(0, T ; H) \text {, } \\
& \tilde{\tau}_{m} \longrightarrow \tau_{*} \quad * \text {-weakly in } L_{\infty}\left(0, T ; L_{2}\right) \text {, } \\
& \left.\left.\tilde{u}_{m}\right|_{\Omega_{k}} \longrightarrow u_{*}\right|_{\Omega_{k}} \text { strongly in } L_{2}\left(0, T ; L_{2}\left(\Omega_{k}\right)\right) \text {, }
\end{aligned}
$$

for every $k$ (there is no strong convergence of $\tilde{\tau}_{m}$ now, because estimate (3.28) depends on $\varepsilon$ ). Obviously, $\left(u_{*}, \tau_{*}\right)$ satisfies estimate (4.3). 
Take arbitrary $\varphi \in \mathscr{V}, \Phi \in C_{0}^{\infty}$. Fix $k$ large enough such that the supports of $\varphi$ and $\Phi$ are contained in $\Omega_{k}$.

Substitute $\left(u_{m}, \tau_{m}\right)$ in equalities (4.1) with $m \geq k, \varepsilon=1 / m$. Take the scalar product of these equalities in $L_{2}(0, T)$ with a smooth scalar function $\psi(t), \psi(T)=0$, and integrate by parts the first terms. Because of the choice of $k$, we can replace $u_{m}$ and $\tau_{m}$ in these equalities by $\tilde{u}_{m}$ and $\tilde{\tau}_{m}$. We have

$$
\begin{aligned}
& -\int_{0}^{T}\left(\tilde{\tau}_{m}, \Phi \varphi^{\prime}(t)\right) d t+\int_{0}^{T}\left(\frac{1}{\lambda_{1}}\left(\tilde{\tau}_{m}, \psi \Phi\right)-\sum_{i=1}^{n}\left(\left(\tilde{u}_{m}\right)_{i} \tilde{\tau}_{m}, \psi \frac{\partial \Phi}{\partial x_{i}}\right)\right. \\
& \left.\quad+2 \mu_{2}\left(\tilde{u}_{m}, \psi \operatorname{Div} \Phi\right)+\frac{1}{\lambda_{1} n}\left(\nabla \tilde{\tau}_{m}, \psi \nabla \Phi\right)\right) d t=\left(\tau_{0}, \Phi\right) \psi(0), \\
& -\int_{0}^{T}\left(\tilde{u}_{m}, \varphi \psi^{\prime}(t)\right) d t+\int_{0}^{T}\left(\mu_{1}\left(\nabla \tilde{u}_{m}, \psi \nabla \varphi\right)-\sum_{i=1}^{n}\left(\left(\tilde{u}_{m}\right)_{i} \tilde{u}_{m}, \psi \frac{\partial \varphi}{\partial x_{i}}\right)+\left(\tilde{\tau}_{m}, \psi \nabla \varphi\right)\right) d t \\
& =\int_{0}^{T}\langle f, \varphi \psi\rangle d t+\left(a_{m}, \varphi\right) \psi(0) .
\end{aligned}
$$

Observe that

$$
\begin{aligned}
\left|\frac{1}{m} \int_{0}^{T}\left(\nabla \tilde{\tau}_{m}, \psi \nabla \Phi\right)\right| & =\left|\frac{1}{m} \int_{0}^{T}\left(\tilde{\tau}_{m}, \psi \Delta \Phi\right)\right| \\
& \leq \frac{1}{m}\left\|\tau_{m}\right\|_{L_{\infty}\left(0, T ; L_{2}\right)} \int_{0}^{T}\|\psi \Delta \Phi\| \underset{m \rightarrow \infty}{\longrightarrow} 0
\end{aligned}
$$

where $\Delta$ is the Laplacian.

Now, we show that

$$
\begin{array}{r}
\int_{0}^{T} \sum_{i=1}^{n}\left(\left(\tilde{u}_{m}\right)_{i} \tilde{\tau}_{m}, \psi \frac{\partial \Phi}{\partial x_{i}}\right) d t \longrightarrow \int_{0}^{T} \sum_{i=1}^{n}\left(\left(\tilde{u}_{*}\right)_{i} \tilde{\tau}_{*}, \psi \frac{\partial \Phi}{\partial x_{i}}\right) d t \\
\int_{0}^{T} \sum_{i=1}^{n}\left(\left(\tilde{u}_{m}\right)_{i} \tilde{u}_{m}, \psi \frac{\partial \varphi}{\partial x_{i}}\right) d t \longrightarrow \int_{0}^{T} \sum_{i=1}^{n}\left(\left(\tilde{u}_{*}\right)_{i} \tilde{u}_{*}, \psi \frac{\partial \varphi}{\partial x_{i}}\right) d t .
\end{array}
$$

Really, using Hölder's inequality, we see that

$$
\begin{aligned}
& \left|\int_{0}^{T} \sum_{i=1}^{n}\left(\left(\tilde{u}_{m}\right)_{i} \tilde{\tau}_{m}-\left(\tilde{u}_{*}\right)_{i} \tilde{\tau}_{*}, \psi \frac{\partial \Phi}{\partial x_{i}}\right) d t\right| \\
& \leq\left|\int_{0}^{T} \sum_{i=1}^{n}\left(\left(\left(\tilde{u}_{m}\right)_{i}-\left(\tilde{u}_{*}\right)_{i}\right) \tilde{\tau}_{m}, \psi \frac{\partial \Phi}{\partial x_{i}}\right) d t\right|+\left|\int_{0}^{T} \sum_{i=1}^{n}\left(\left(\tilde{u}_{*}\right)_{i}\left(\tilde{\tau}_{m}-\tilde{\tau}_{*}\right), \psi \frac{\partial \Phi}{\partial x_{i}}\right) d t\right| \\
& \leq\left.\left\|\left.\left.\left(u_{m}-u_{*}\right)\right|_{\Omega_{k}}\right|_{L_{2}\left(0, T ; L_{2}\right)}|| \tau_{m}\right\|_{L_{2}\left(0, T ; L_{2}\right)}|| \psi \frac{\partial \Phi}{\partial x_{1}}\right|_{L_{\infty}\left(0, T ; L_{\infty}\right)}+\left\langle\tilde{\tau}_{m}-\tilde{\tau}_{*}, \sum_{i=1}^{n}\left(\tilde{u}_{*}\right)_{i} \psi \frac{\partial \Phi}{\partial x_{1}}\right\rangle .
\end{aligned}
$$


The last brackets denote the action of the functional $\tilde{\tau}_{m}-\tilde{\tau}_{*}$ from $L_{1}\left(0, T ; L_{2}\right)^{*}=L_{\infty}(0, T$; $\left.L_{2}\right)$ on the element $\sum_{i=0}^{n}\left(\tilde{u}_{*}\right)_{i} \psi\left(\partial \Phi / \partial x_{i}\right)$. Both terms tend to zero, and (5.7) is proved. Similarly one shows (5.8).

Now, let $m$ tend to infinity in (5.5). We obtain

$$
\begin{aligned}
& -\int_{0}^{T}\left(\tau_{*}, \Phi \psi^{\prime}(t)\right) d t+\int_{0}^{T}\left(\frac{1}{\lambda_{1}}\left(\tau_{*}, \psi \Phi\right)-\sum_{i=1}^{n}\left(\left(u_{*}\right)_{i} \tau_{*}, \psi \frac{\partial \Phi}{\partial x_{i}}\right)+2 \mu_{2}\left(u_{*}, \psi \operatorname{Div} \Phi\right)\right) d t \\
& =\left(\tau_{0}, \Phi\right) \psi(0), \\
& -\int_{0}^{T}\left(u_{*}, \varphi \psi^{\prime}(t)\right) d t+\int_{0}^{T}\left(\mu_{1}\left(\nabla u_{*}, \psi \nabla \varphi\right)-\sum_{i=1}^{n}\left(\left(u_{*}\right)_{i} u_{*}, \psi \frac{\partial \varphi}{\partial x_{i}}\right)+\left(\tau_{*}, \psi \nabla \varphi\right)\right) d t \\
& =\int_{0}^{T}\langle f, \varphi \psi\rangle d t+(a, \varphi) \psi(0) .
\end{aligned}
$$

As in the proof of Theorem 4.1, it follows from here that $\left(u_{*}, \tau_{*}\right)$ is a solution of (3.1), (3.2), (3.5) and $u_{*}$ satisfies estimate (3.27). To prove (5.2), it remains to estimate the fifth term in its left part.

Substitute $\left(u_{*}, \tau_{*}\right)$ in (3.1). Using Hölder's inequality, the embedding $V \subset L_{4}$, and (4.3), we have

$$
\begin{aligned}
\left\|\frac{d}{d t}\left(\tau_{*}, \Phi\right)\right\|_{L_{2}(0, T)} \leq & \left\|\frac{1}{\lambda_{1}}\left(\tau_{*}, \Phi\right)\right\|_{L_{2}(0, T)}+\left\|\sum_{i=1}^{n}\left(\left(u_{*}\right)_{i} \tau_{*}, \frac{\partial \Phi}{\partial x_{i}}\right)\right\|_{L_{2}(0, T)} \\
& +\left\|2 \mu_{2}\left(u_{*}, \operatorname{Div} \Phi\right)\right\|_{L_{2}(0, T)} \\
\leq & \frac{1}{\lambda_{1}}\left\|\tau_{*}\right\|_{L_{2}\left(0, T ; L_{2}\right)}\|\Phi\|_{L_{2}}+\left\|u_{*}\right\|_{L_{2}\left(0, T ; L_{4}\right)}\left\|\tau_{*}\right\|_{L_{\infty}\left(0, T ; L_{2}\right)}\left\|\frac{\partial \Phi}{\partial x_{i}}\right\|_{L_{4}} \\
& +2 \mu_{2}\left\|u_{*}\right\|_{L_{2}\left(0, T ; L_{2}\right)}\|\operatorname{Div} \Phi\|_{L_{2}} \\
\leq & C\|\Phi\|_{H_{0}^{2}}\left(\left\|\tau_{*}\right\|_{L_{\infty}\left(0, T ; L_{2}\right)}+\left\|u_{*}\right\|_{L_{2}(0, T ; V)}\left\|\tau_{*}\right\|_{L_{\infty}\left(0, T ; L_{2}\right)}+\left\|u_{*}\right\|_{L_{2}(0, T ; V)}\right) \\
\leq & K_{4}\left(\|a\|,\left\|\tau_{0}\right\|,\|f\|_{L_{2}\left(0, T ; V^{*}\right)}\right)\|\Phi\|_{H_{0}^{2} .}
\end{aligned}
$$

Taking into account (3.12), we obtain the desirable estimate.

By [10, Chapter III, Lemma 1.1] a.e. in $(0, T) u_{*}$ is equal to a continuous function on $[0, T]$ with values in $V^{*}$. Without loss of generality we may assume that $u_{*}$ itself is continuous on $[0, T]$ with values in this space. And since it belongs to $L_{\infty}(0, T ; H)$, it is weakly continuous on $[0, T]$ with values in $H$ (see [10, Chapter III, Lemma 1.4]). Similarly $\tau \in C_{w}\left([0, T] ; L_{2}\right)$. The proof of the theorem is complete.

Remark 5.2. By the same scheme it is easy to show that in Theorems 3.2 and 4.1 the condition of boundedness of $\Omega$ is not necessary. 
Proof of Theorem 2.3. Take $\tau_{0}=\sigma_{0}-2 \mu_{1} \mathscr{E}(a)$. By the condition of the theorem $\tau_{0} \in L_{2}$. By Theorem 5.1 there exists a solution $(u, \tau)$ of problem (3.1), (3.2), (3.5) in class (5.1). It is easy to see that $\mathscr{E}(u) \in L_{2}\left(0, T ; L_{2}\right) \cap C_{w}\left([0, T] ; H^{-1}\right)$.

Take $\sigma=\tau+2 \mu_{1} \mathscr{E}(u)$. Then the pair $(u, \sigma)$ belongs to class (2.10) and satisfies (2.5), (2.11), that is, it is a weak solution of problem (2.1)-(2.5).

\section{Acknowledgment}

This work was partially supported by Grants 01-01-00425 of the Russian Foundation of Basic Research and VZ-010-0 of the Ministry of Education of Russia and CRDF.

\section{References}

[1] Y. Y. Agranovich and P. E. Sobolevskiǔ, Motion of a nonlinear viscoelastic fluid, Dokl. Akad. Nauk SSSR 314 (1990), no. 3, 521-525 (Russian).

[2] V. T. Dmitrienko and V. G. Zvyagin, The topological degree method for equations of the NavierStokes type, Abstr. Appl. Anal. 2 (1997), no. 1-2, 1-45.

[3] H. Gajewski, K. Gröger, and K. Zacharias, Nichtlineare Operatorgleichungen und Operatordifferentialgleichungen, Akademie-Verlag, Berlin, 1974.

[4] C. Guillopé and J.-C. Saut, Mathematical problems arising in differential models for viscoelastic fluids, Mathematical Topics in Fluid Mechanics (Lisbon, 1991) (J. F. Rodrigues and A. Sequeira, eds.), Pitman Res. Notes Math. Ser., vol. 274, Longman Scientific Technology, Harlow, 1992, pp. 64-92.

[5] I. Gyarmati, Non-Equilibrium Thermodynamics. Field Theory and Variational Principles, Springer, Heidelberg, 1970.

[6] A. A. Kotsiolis and A. P. Oskolkov, Solvability of the fundamental initial-boundary value problem for the equations of motion of Oldroyd fluids on $(0, \infty)$ and the behavior of its solution as $t \rightarrow+\infty$, Zap. Nauchn. Sem. Leningrad. Otdel. Mat. Inst. Steklov. (LOMI) 150 (1986), 4852 (Russian).

[7] V. G. Lìtvīnov, Dvizhenie Nelineino-Vyazkoi Zhidkosti [Motion of a Nonlinearly Viscous Fluid], Nauka, Moscow, 1982.

[8] J. G. Oldroyd, Non-Newtonian flow of fluids and solids, Rheology: Theory and Applications, Inostrannaya Literatura, Moscow, 1962, pp. 757-793.

[9] M. Reiner, Rheology, Fizmatgiz, Moscow, 1965.

[10] R. Temam, Navier-Stokes Equations, Studies in Mathematics and Its Applications, vol. 2, NorthHolland Publishing, Amsterdam, 1979.

[11] V. G. Zvyagin and V. T. Dmitrienko, On weak solutions of a regularized model of a viscoelastic fluid, Differ. Uravn. 38 (2002), no. 12, 1633-1645 (Russian).

D. A. Vorotnikov: Department of Mathematics, Voronezh State University, Universitetskaya Pl. 1, Voronezh 394006, Russia

E-mail address: mitvorot@math.vsu.ru

V. G. Zvyagin: Department of Mathematics, Voronezh State University, Universitetskaya Pl. 1, Voronezh 394006, Russia

E-mail address: zvg@main.vsu.ru 


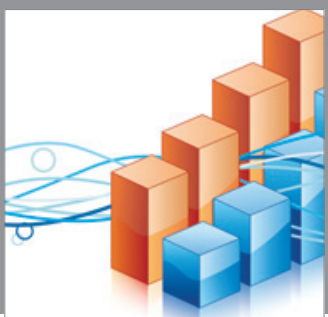

Advances in

Operations Research

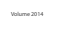

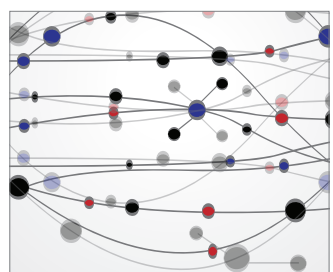

\section{The Scientific} World Journal
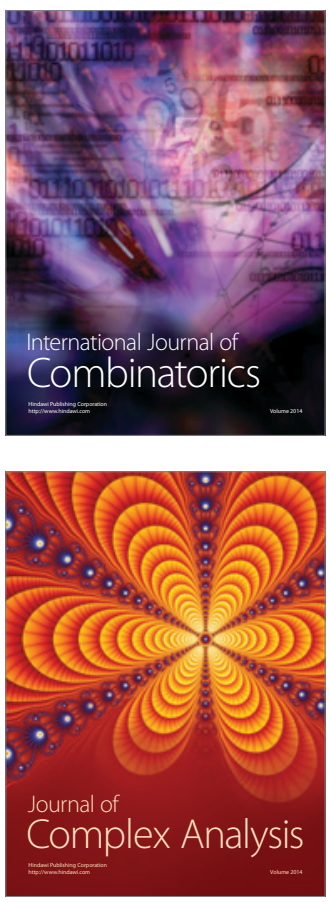

International Journal of

Mathematics and

Mathematical

Sciences
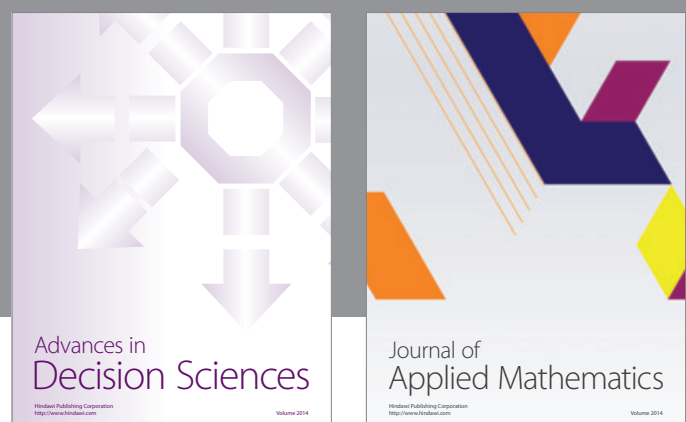

Journal of

Applied Mathematics
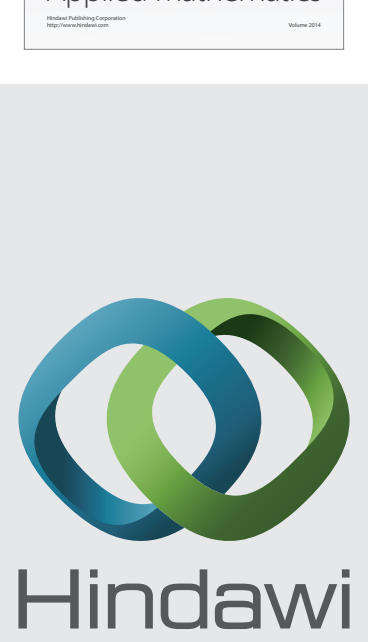

Submit your manuscripts at http://www.hindawi.com
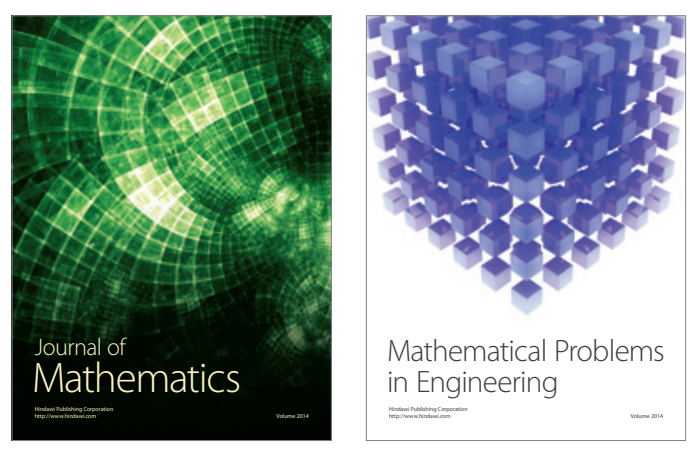

Mathematical Problems in Engineering
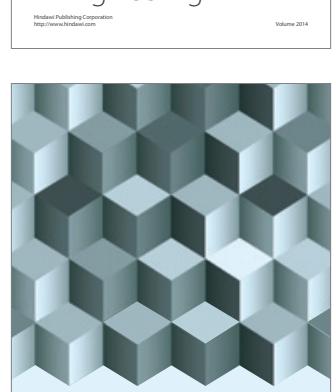

Journal of

Function Spaces
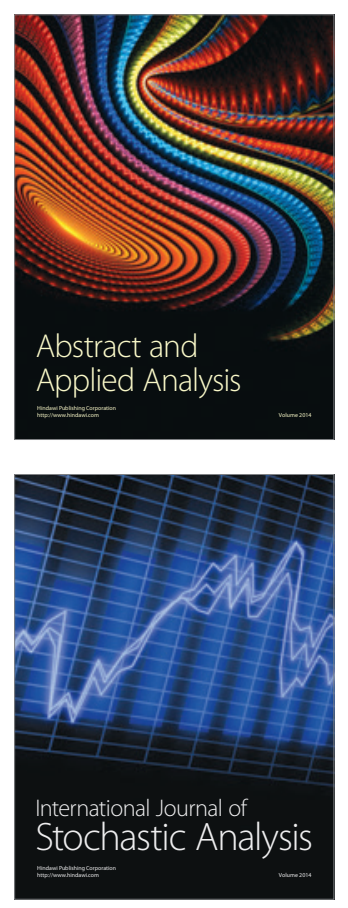

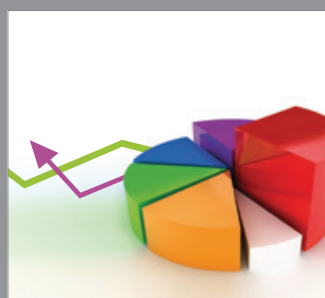

ournal of

Probability and Statistics

Promensencen
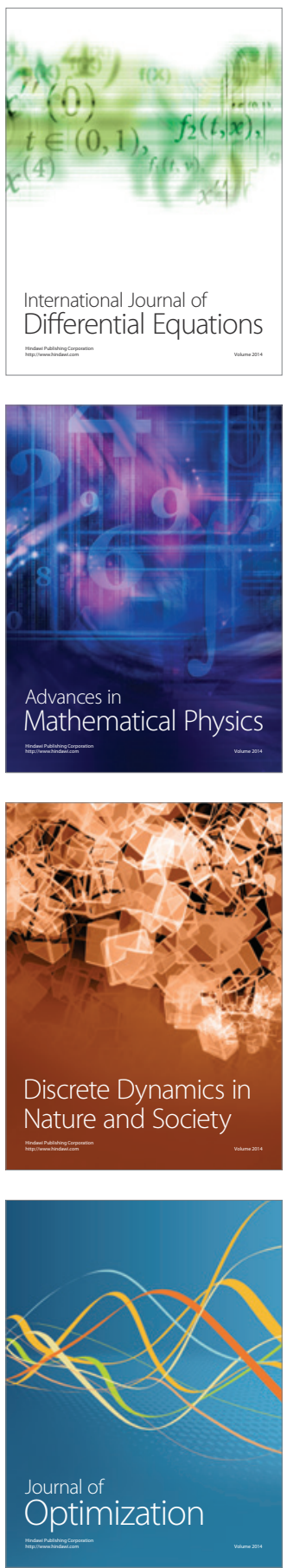